\title{
Advances in Switched-Mode Power Conversion Part I
}

\author{
SLOBODAN ĆUK, MEMBER, IEEE, AND R. D. MIDDLEBROOK, FELLOW, IEEE
}

\begin{abstract}
A new concept in the design of switched-mode power conversion circuitry is presented. Because of its extreme simplicity, flexibility, and efficiency, it has the potential to replace some conventional electrical power processing methods currently in use. A thorough discussion of the background, development, and operation of the Cuk converter and several of its extensions is included. The coupled-inductor technique further improves efficiency and reduces size and weight. The basic converter and its extensions to twoquadrant and four-quadrant operation are attractive for many applications, i.e., solar (dc) to utility line (ac) power conversion, variable speed dc or ac motor drives, switching power supplies, uninterruptible power supplies, and many others.
\end{abstract}

\section{INTRODUCTION}

$\mathbf{S}^{\mathrm{m}}$ WITCHING POWER supplies and regulators ("switchers") have come into widespread use in the last decade. Because of their much higher efficiency, smaller size and weight, and relatively low cost, they are displacing conventional linear power supplies, even at low power levels (about $25 \mathrm{~W}$ ). The design of switching converters has been extensively studied, and it is commonly believed that the designs in commercial use today employ the simplest possible switching structures for dc-to-dc level conversion.

However, even though high conversion efficiency has been achieved, present switching converter designs possess several desirable characteristics. Rapid switching of the input or output currents can cause severe electromagnetic interference (EMI) problems, requiring the addition of appropriate filters that increase both the complexity and cost of the circuit. Also, the implementation of the power transistor switch requires complex drive circuitry in configurations where the emitter voltage "floats" above ground. In switched-mode power amplifier designs, additional problems are imposed by the requirements for dual (bipolar) power supplies, high switching frequencies, and complex feedback circuitry.

A revised look at present converter designs and an analysis of switching circuit topology has led to the discovery of a new design that retains all the desirable properties of conventional designs, with none of their undesirable attributes. Both input and output currents are essentially nonpulsating $\mathrm{dc}$, and, in fact, ripple can be reduced to zero. Furthermore, the new topology may be implemented with fewer parts than comparable solutions, and thus may be said to be an "optimal" design.

Because of its simplicity and generality, the new converter

Manuscript received October 15,1982 . This article is reprinted from Robotics Age Magazine.

The authors are with the California Institute of Technology, Pasadena, CA 91125. can efficiently raise or lower dc levels, and, with minor modification, bidirectional power flow can be easily achieved, allowing the roles of power source and load to be arbitrarily interchanged without physically switching their connections. A switching power amplifier based on the new converter requires only a single power supply, enabling efficient dc-to-ac power conversion. The improved performance of the new switching power amplifier design permits the use of a lower switching frequency and simple circuits for both the drive and feedback.

We will begin with a discussion of switching converter design, leading to the development of the new converter. Next, the design of a switched-mode power amplifier using the new converter and a discussion of its performance will be presented.

\section{A. A Review of Switching Converter Design}

In the basic design of the linear power supply shown in Fig. 1 , the output voltage $V_{o}$ is regulated to a multiple of the reference voltage $V_{\text {ref. }}$. The difference between the unregulated input voltage and $V_{o}$ results in power dissipation in the transistor, which, in high current supplies, can result in considerable energy loss and heating. Consequently, the unregulated voltage must be kept as low as possible while still allowing adequate regulation.

Switching power supplies are based on the principle that, by alternately switching the transistor completely off and on, its power dissipation can be held to a minimum. Passive energy storage elements, inductors, and capacitors can then be used to transfer energy from the source to the load, performing the appropriate level conversion in the process. ${ }^{1}$

Fig. 2 shows the most commonly used switching configuration, referred to as the "buck" converter. The ideal switch $S$ can be realized by the combination of bipolar transistor and commutating diode as shown. In operation, the input voltage is connected by $S$ to charge inductor $L$ to the output current necessary to produce the desired voltage $V$ across load $R$. Once $V$ is attained, $S$ disconnects the input and provides an alternate path for the inductor current, which then begins to decay. The output capacitor $C$ helps reduce the residual voltage ripple caused by the switching. The cycle repeats at a fixed rate, and the average voltage gain is equal to the duty ratio $D$ (the frac-

1 Recall from basic physics that energy is stored by an inductor in the magnetic field produced by the current through its winding. A voltage applied across it either increases or decreases this current to energize or deenergize the field. Conversely, the current through a capacitor either charges or discharges the energy stored in an electric field, respectively raising or lowering the voltage across its terminals. 


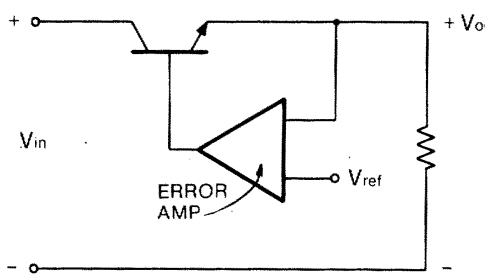

Fig. 1. Conventional linear power conversion.

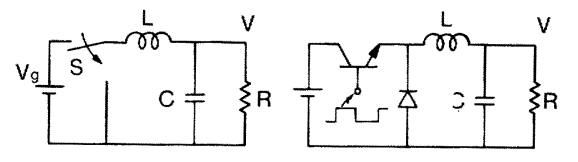

(a)

(b)

Fig. 2. The basic "buck"-type switching converter configuration, showing (a) the topology of the circuit, using an idealized switch $S$, and (b) the implementation of the switch using a bipolar transistor and a commutating diode.

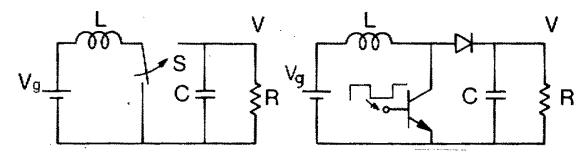

(a)

(b)

Fig. 3. The common "boost" converter configuration. (a) Circuit topology, using an ideal switch, and (b) implementation.

tion of the switching cycle that the transistor is on).

Note that the input current to the regulator alternates between the full output current and zero. This abrupt variation in the input energy flow causes severe EMI, and invariably requires the presence of an input filter to smooth out the sub. stantial current ripple component at the switching frequency. Also, since the emitter floats above ground, isolated drive circuitry (not shown) is required to switch the transistor. A further limitation of this design is that only voltage reduction is possible, since $D$ is less than 1 .

For applications requiring a voltage step-up, some of the drawbacks of the buck configuration can be avoided by using the "boost" converter configuration, shown in Fig. 3. In this design, the inductor is always in series with the input, so that the supply current is continuous. The inductor stores energy when grounded by $S$, and then the stored energy is released to the output. The output voltage of the boost converter is always greater than the supply, with an ideal gain equal to $1 /(1-$ $D$ ). Note also that the grounded emitter of the transistor switch simplifies the drive circuit. The drawback of the configuration, however, is that during the inductor charging interval, all the output current must be supplied by discharging the output capacitor, resulting in considerable output ripple.

\section{DEVELOPMENT OF THE NEW CONVERTER TOPOLOGY}

The discovery of the new converter design resulted from the objective of retaining the desirable properties of both types of converters. This goal was realized by examining combinations of the two basic types, with the underlying princi-

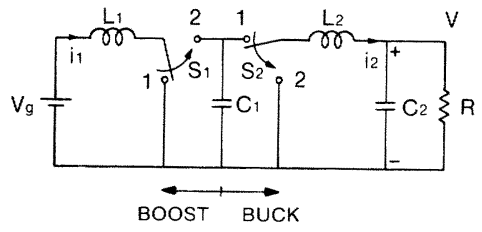

Fig. 4. Cascade combination of a boost and a buck power stage.

ples of simplification and optimum interconnection while simultaneously maximizing performance. The details of the analytical technique, described more completely in [1], will be abbreviated here.

Consider the cascade connection of a boost power stage followed by a buck power stage, resulting in the converter shown in Fig. 4. This configuration retains the desirable properties of the low input current ripple of the boost converter and the low output current ripple of the buck stage. The voltage gain of the converter is the product of the gains of the two stages. Assuming that $S_{1}$ and $S_{2}$ are synchronized so that both switch from position 1 to 2 (and back) simultaneously, the resulting ideal gain is $D / D^{\prime}$, where $D^{\prime}=1-D$. Thus, the same converter can be used for both level reduction $(D<0.5)$ and increase $(D>0.5)$.

The undesirable output ripple of the boost converter is now isolated between the stages, and, in fact, the capacitor serves as the sole energy transfer mechanism between the stages. To see this, note that during the part of the cycle when both switches are in position 2, $C_{1}$ is isolated from the output circuit and is charged by the input current through $L_{1}$. In the rest of the cycle, $C_{1}$ is completely transferred to the output circuit and is discharged by the output current through $L_{2}$, reenergizing $L_{2}$ while $L_{1}$ stores energy from the input. Position 2 of $S_{2}$ provides a path to sustain the output current (from energy in $L_{2}$ ) while $C_{1}$ is being recharged by $L_{1}$.

The issue remains, however, as to whether or not this design represents the optimal configuration of this attractive boost-buck cascade. It is apparent that the two inductors are essential to the continuity of the input and output currents, and that the intermediate capacitor is required for energy transfer. The issue of optimality thus resolves to the questions, can the number of switches in this cascade configuration be reduced from two to one and still achieve capacitive energy transfer?

The answer to this question may at first seem surprising. Switches $S_{1}$ and $S_{2}$ may indeed be combined, resulting in a new optimal converter configuration. The solution is found by considering the topological properties of known converter types and their cascaded combinations [1], [2] . A key to the solution is that inversion of the converter's output voltage is a necessary feature of the new design. Since both of the basic converter types are noninverting, the only way to achieve this is by reversing the polarity of the charged energy transfer capacitor when it is switched into the output circuit. Attention to this issue alone leads to the solution shown in Fig. 5.

Even though a true dc-to-dc transformer is physically impossible, the new converter can functionally be considered as such, since both its input and output voltages and currents are very close to true dc quantities, owing to the negligible switch- 


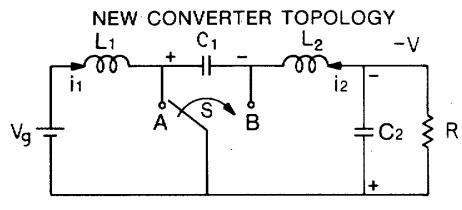

Fig. 5. The new switching topology, employing capacitive energy transfer with polarity inversion of the output voltage.

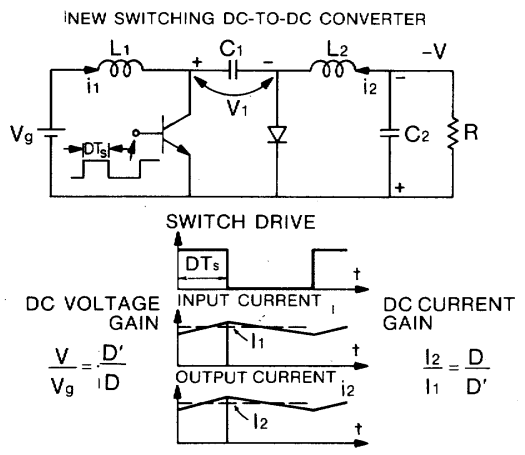

Fig. 6. Hardware realization of the Cuk converter using a transistor and a diode to implement switch $S$. Ideal dc voltage and current gains are as shown.

ing ripple. Moreover, due to the advantages of capacitive energy transfer, the actual conversion efficiency of the new circuit is substantially greater than that of conventional designs [1]. The grounded emitter of the switching transistor also allows the most simple drive circuitry to be used.

In this design, the single switch $S$ alternately grounds the opposite ends of the capacitor, effectively switching it from the input to the output circuits. $C_{1}$ is charged by the input current to a positive voltage, as viewed from left to right, with $S$ in position $B$. With $S$ switched to $A$ (during the charging interval of $L_{1}$ ), the "positive" side of $C_{1}$ is now connected to ground, and its "negative" side (since the voltage across it does not change) effectively "pulls down" the voltage level at terminal $B$. Thus, current flows from the grounded load to discharge $C_{1}$ through $L_{2}$, causing a negative voltage drop across the load, hence a negative output voltage.

The hardware implementation of the new converter, using a transistor/diode combination to implement the ideal switch $S$ as in the other converter configurations, is shown in Fig. 6. With the transistor off (open), $C_{\mathbf{1}}$ is charged by the input current through the forward biased diode. When the transistor turns on, it provides a charging path for $L_{1}$, but also drops the positive terminal of $C_{1}$ to (very near) ground. As in the ideal case, this pulls down the output voltage, reversing the bias on the diode (turning it off) and transferring the stored energy to $L_{2}$ in the form of increased current. Note that the dual role of switch $S$, its inclusion in the input and output circuits simultaneously, requires that the transistor and the diode, when conducting, must carry both the input and the output currents.

\section{A. Coupled Inductor Extension of the New Converter}

It would seem from the above discussion that the simplest possible converter circuit has been obtained, but this is not the case. Consideration of the voltage waveforms across the two inductors $L_{1}$ and $L_{2}$ over the switching cycle reveals that, for the average dc voltage across each inductor to be zero (steady-

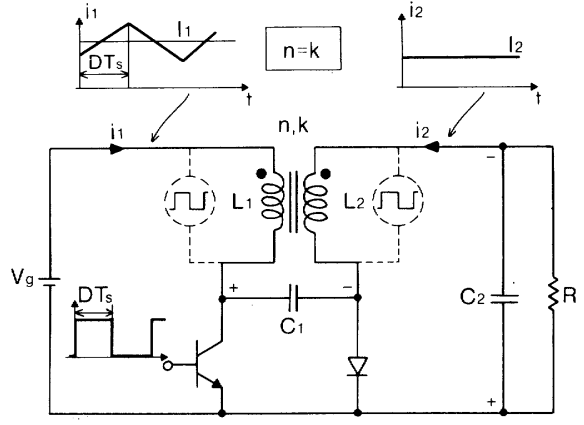

Fig. 7. The Coupled Inductor Extension of the Cuk converter. With appropriate transformer design, the output current ripple can be reduced to zero.

state balance condition), the two waveforms must be identical [3]. This means that the two inductors may be coupled by being wound on the same core, without affecting the basic $\mathrm{dc}$ conversion property, provided that the resulting transformer has a $1: 1$ primary to secondary voltage ratio. This is easily achieved by setting $L_{1}=L_{2}$ (same number of turns in each), with the direction of coupling as shown in Fig. 7.

The resulting circuit not only eliminates one inductor core (for further component savings), but has an interesting property that profoundly affects the performance of the new converter. Since the coupled inductors form a transformer, inductive energy transfer between the two windings alters the effective inductance of each. For a 1:1 turns ratio, both inductances are approximately doubled, so that both input and output current ripples are about one half those of the uncoupled converter. Even more significant, however, is that by slightly altering the turns ratio, one of the effective inductance values can be made arbitarily large, dramatically reducing the current ripple on that side. When the effective turns ratio (primary to secondary) matches the inductive coupling coefficient of the transformer, ${ }^{2}$ the output current ripple can be completely eliminated, resulting in pure dc to the load [3]. In this matching condition, no output capacitor is needed, since the load voltage is also constant.

The coupled-inductor converter thus has the simplest possible structure, consisting of a single transformer, commutation capacitor, and a single switch (realized by two semiconductors), and yet it achieves the maximum performance (both input and output current nonpulsating with one of them even being pure dc with no ripple) in a topology which offers the smallest possible size and weight and highest efficiency.

It is interesting to note that the idea of coupling the in ductors is a new concept which can be implemented with similar benefits to many other switching converter configurations. Nevertheless, the greatest potential of this configuration is obtained through its application to the new converter topology, on which it was originally conceived.

Yet, this still seems to fall short of the ultimate objective, the functional realization of the ideal dc-to-dc transformer shown schematically in Fig. 8, which has constant current at both input and output. In addition, the dc isolation between

2 Like the effective turns ratio, the inductive coupling coefficient is a physical property of the transformer construction, with a value ranging from 0 (separate inductors) to very near 1 (windings closely wound on a single ferromagnetic core). 


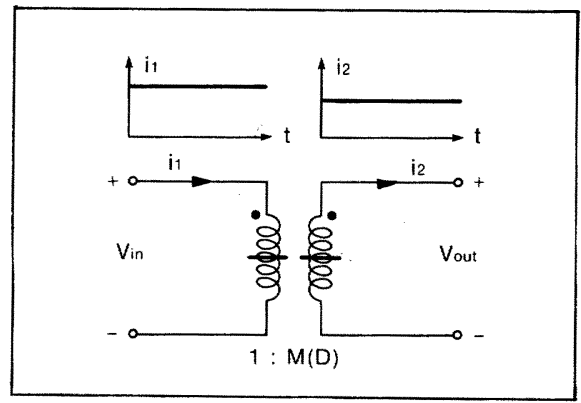

Fig. 8. The ultimate objective of switching conversion-A dc-to-dc transformer with constant input and output currents and an electronically controllable effective turns ratio for voltage level change.

input and output shown in Fig. 8, often required in many practical applications, is not present in any of the converter extensions discussed so far. This, however, poses no insurmountable problems, since there is an elegant way of introducing dc isolation into the new converter by the addition of a single ac transformer and an extra capacitor, as described in detail in [4]. Furthermore, the same idea of coupling inductors in this dc-isolated extension leads also to zero current ripple on one side.

The latest generalization of the new coupled-inductor converter concept has resulted in what may be considered the ultimate solution: a dc-isolated switching dc-to-dc converter with zero current ripple at both input and output terminals, which functionally emulates the ideal dc-to-dc transformer of Fig. $8 .^{3}$

\section{B. Bidirectional Power Flow in the New Converter}

Note that the configuration of the new converter shown in Fig. 5 is completely symmetrical with respect to the designation of the input and output terminals. Because the ideal switch $S$ allows current flow in either direction, either terminal of the converter can behave as a current source or as a current sink. However, when the switch is implemented by a single transistor and diode, only unidirectional current flow is allowed. The addition of a single p-n-p transistor and a diode removes this constraint and results in bidirectional current and power flow as shown in Fig. 9.

The converter circuit is thus symmetrical, and the input and output terminals can be arbitrarily designated (as long as the voltage polarities are respected). The configuration shown in Fig. 9 is ideal for battery charger/discharger applications, since both functions are accomplished by this single converter circuit [5]. The direction of power flow through the converter is determined by whether the duty ratio is greater or less than the value that gives a voltage gain equal to the ratio of the bus to battery voltages. In general, attempting to reduce the voltage to a load that stores energy causes power to be transferred from the load, ${ }^{4}$ just as attempting to raise the voltage across

3 Technical details of this latest development have not yet been publicly released, but are contained in a patent application. The basic Cuk converter configuration and many of its improvements, as well as new switching power amplifier configurations, are protected by a series of patents [9]-[12].

4 Editor's note: A very useful application of this is to use a de drive motor as a generator for regenerative braking, recovering some of the energy stored in a robot's momentum or provided by an external source (as when rolling down a slope).

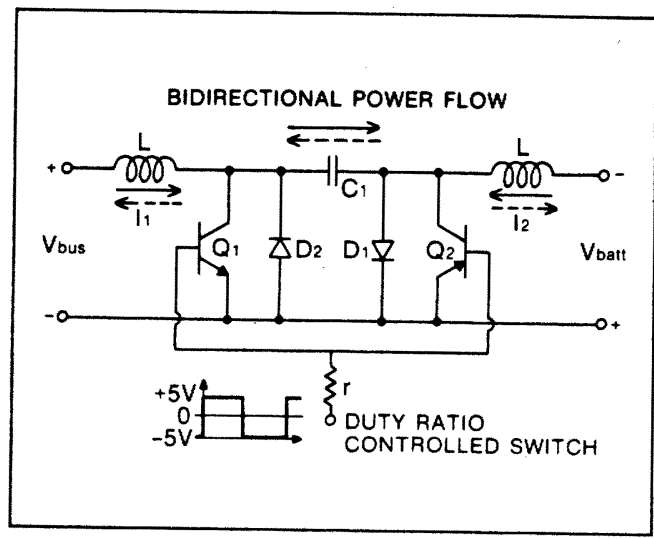

Fig. 9. A symmetrical implementation of the Cuk converter, capable of power transfer in either direction.

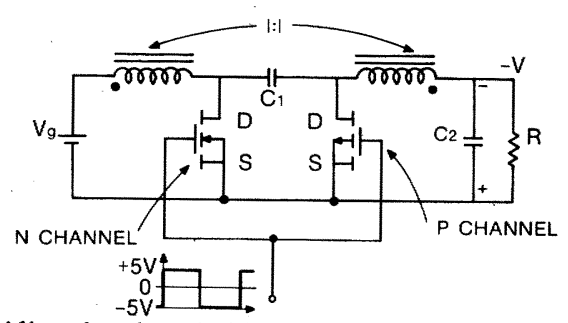

Fig. 10. Bidirectional switching converter using MOSFET power switches.

a load normally requires the transfer of power to the load. However, when two sources of stored energy are coupled by a bidirectional converter, precise control of the duty ratio is essential to restrict the resulting power flow to an acceptable limit. The bidirectional current switch implementation is equally applicable to the coupled-inductor extension (Fig. 7) of the new converter.

Since both of the transistors in Fig. 9 are referenced to ground, the complementary switching of the pair can be accomplished by a single drive source as shown. Moreover, because the two base junctions are tied together, the circuit also automatically prevents the simultaneous turnon of both transistors (and thus prevents shorting out capacitor $C$ ), in spite of the presence of transistor switch storage time.

Another implementatation of the bidirectional current switch is made possible by recent technological advances in metal oxide semiconductor field effect transistors (MOSFET's). Formerly limited mostly to small signal applications, newer MOSFET devices are capable of switching to a relatively low ON resistance, with a substantially higher current rating. It is not widely known that the power MOSFET's are capable of bidirectional current flow. Owing to the device's internal construction, there is effectively an inherent diode connected between drain and source which provides the alternate (opposing) current path. Hence, each transistor/diode pair can be replaced by a single power MOSFET device, resulting in the bidirectional converter shown in Fig. 10. If both p-channel and n-channel MOSFET's are used, the source terminals of both devices may be grounded, allowing a simple driving scheme similar to that used for the grounded emitter transistors in Fig. 9.

Besides reducing the component count, power MOSFET's have a number of advantages over bipolar transistors which make them especially attractive for switching converter appli- 
cations [6]. Whereas bipolars are current-controlled devices, FET's are voltage controlled. As a consequence, they require much lower drive currents and are capable of very fast switching speeds. Also, unlike bipolars, several MOSFET's can be easily paralleled to distribute switching current. Their switching speed ( 5 ns typical) is one or two orders of magnitude faster than comparable bipolars (200 ns typical), significantly reducing power loss during switching. Although older power MOSFET's had a high saturation voltage compared to bipolars ( $3 \mathrm{~V}$ at $10 \mathrm{~A}$ versus $2 \mathrm{~V}$ or less for a bipolar), the newest devices have as little as $0.055 \Omega R_{S D}$, making their use in converter designs extremely attractive, especially for applications requiring a high switching frequency.

\section{APPLICATION OF THE NEW CONVERTER CIRCUIT TO SWITCHED-MODE POWER AMPLIFIER DESIGN}

Although a substantial effort has been made in recent years toward the development of complex switching power supplies, substantially less attention has been devoted to their natural outgrowth-switching power amplifiers. It is quite natural, then, that the principles of the operation of switching amplifiers are not widely known. We will begin, therefore, with a review of present designs, which are all based on a modification of the "buck" power stage of Fig. 2. This review reveals substantial performance deficiencies originating from the buck converter itself.

The new circuit topology of Fig. 5 eliminates most of the problems associated with earlier switching converter designs, providing greatly improved performance with fewer components. This, together with the extension of the new design to allow bidirectional current flow, suggests that the design of switching amplifiers should be reexamined in light of this new development. Indeed, we will show that a power amplifier design based on the new converter topology provides a much superior solution.

\section{A. Switching Amplifier Principles of Operation}

The major difference between the power stages of a switching amplifier and a switching power supply is that the former must be capable of producing an output of either polarity. In all present designs, this is accomplished by modifying the basic buck power stage to use two power supplies $+V_{g}$ and $-V_{g}$ as input, with the switch $S$ switching between positive and negative supplies as shown in Fig. 11.

The resulting voltage gain, shown in Fig. 12, is a linear function of the duty ratio $D$. For $D$ greater than 0.5 , the output voltage is positive, while for $D$ less than 0.5 , it is negative. Note that since the load voltage may be negative as well as positive, the implementation of switch $S$ shown in Fig. 2 is inadequate. Since the output voltage is determined by the inductor current, the hardware implementation of the switch must permit bidirectional current flow, as shown by the arrows in Fig. 11. This is readily accomplished by the twotransistor, two-diode circuit shown in Fig. 13. This bidirectional implementation is similar to that required for the symmetrical converter in Fig. 9, but with the significant difference that neither transistor is referenced to ground, necessitating the use of isolated drive circuitry to accomplish the complementary switching action.

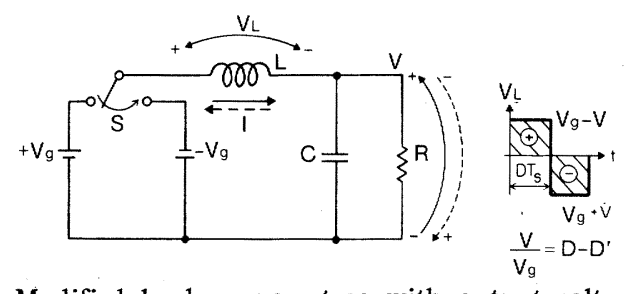

Fig. 11. Modified buck power stage with output voltage of either polarity.

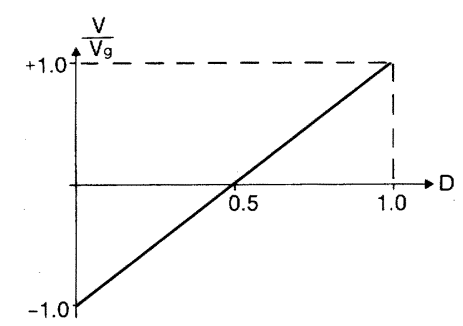

Fig. 12. DC voltage gain of the power stage in Fig. 11.

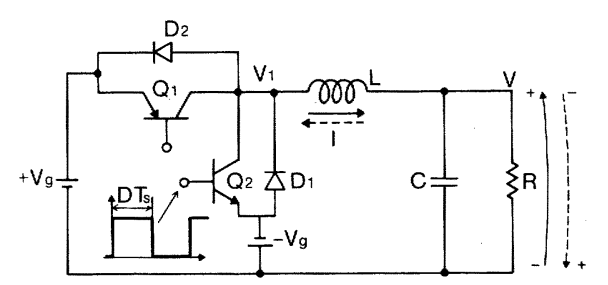

Fig. 13. Practical implementation of the converter in Fig. 11.

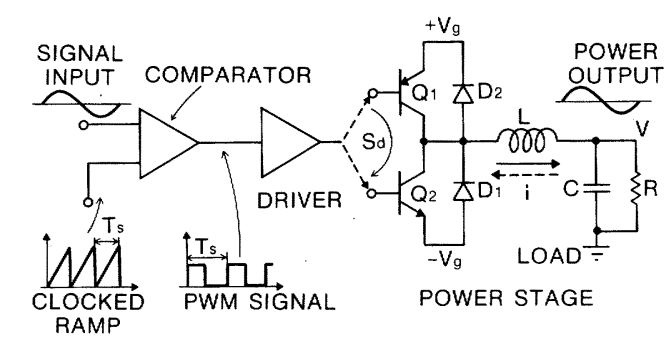

Fig. 14. Open-loop buck-type switching power amplifier.

To apply this dual-polarity power stage in a switching power amplifier, it is necessary to control the duty cycle so that the output voltage varies in proportion to the input signal. The linear voltage gain of the buck power stage facilitates this function, and is incorporated into the open-loop amplifier configuration shown in Fig. 14. The design is the same as that for an open-loop dc-to-dc converter operated at a constant switching frequency $f=1 / T_{s}$, with the only difference that a time varying (sinusoidal, for instance) input signal is used at the comparator input, instead of a dc reference voltage. When the input signal is positive, a pulse with $D$ greater than 0.5 is generated, producing positive output, while for negative input, $D$ is less than 0.5 and negative output is produced. In fact, comparison of the low-frequency input signal and the high-frequency sawtooth (clocked ramp), generates a pulse width modulated (PWM) signal, whose low frequency spectrum is, in effect, recovered by low-pass filtering with the inductor. Hence, a close replica of the input signal is generated at the output, but at a high power level.

The comparison of this switching amplifier approach with conventional linear designs with respect to the two foremost 


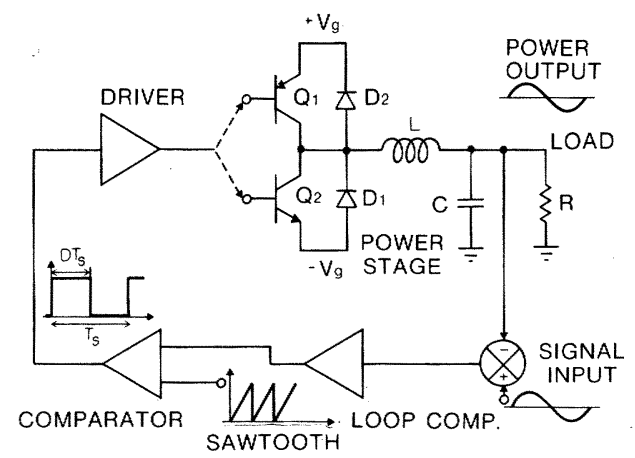

Fig. 15. Closed-loop buck-type switching power amplifier.

constraints in power amplifier design, efficiency, and distortion, now becomes apparent. In terms of efficiency, this approach boasts the usual advantages of switching power supplies over linear-significantly lower power dissipation. Namely, its theoretical 100-percent efficiency is usually only slightly degraded (often it is over 90 percent) by losses due to nonzero transistor saturation voltage and switching time and parasitic resistances of storage elements in the power path.

Distortion, however, becomes a function of the switching frequency, rather than being dependent on the linearity of the transistor gain curve. Specifically, for low distortion, the switching frequency has to be an order of magnitude or so higher than the signal frequency to avoid overlapping sidebands in the PWM signal and to minimize the effects of output ripple. On the other hand, with increased switching frequency, the switching time of the transistor may represent a significant portion of the duty cycle, introducing further distortion and degrading efficiency as well. Other distortion sources arise from nonlinearities in the clocked ramp (which also increases with switching frequency) and variations in the power supply voltages.

To reduce the effects of these sources of distortion requires the use of negative feedback, as shown in the block diagram in Fig. 15. Feedback allows a reduced switching frequency and improves linearity at the expense of increased circuit complexity, but the amplifier in Fig. 15 still has drawbacks that originate directly from the use of the buck power stage. As in the buck power supply design, the input currents pulsate, at the switching frequency, between zero and the output current, causing severe EMI. The design requires two power supplies of opposite polarity, and complex drive circuitry is required for the transistors, both to translate drive signals to the ungrounded emitters, and to prevent simultaneous turn on of the transistors and shorting of the power supplies. Finally, a relatively high switching frequency $(300 \mathrm{kHz}$ or so) is still necessary to reduce switching ripple.

As in the case of power supply design, the introduction of the new switching converter topology solves all of these problems, but the objective of achieving maximum performance (wide bandwidth, low noise and distortion, small (or zero) switching ripple, and low switching frequency), for the minimum number and size of parts, is by no means an easy one.

\section{B. A New Push-Pull Switching Power Amplifier}

We now pose the problem of inventing a power stage, based on the new converter of Fig. 5, which will produce an output

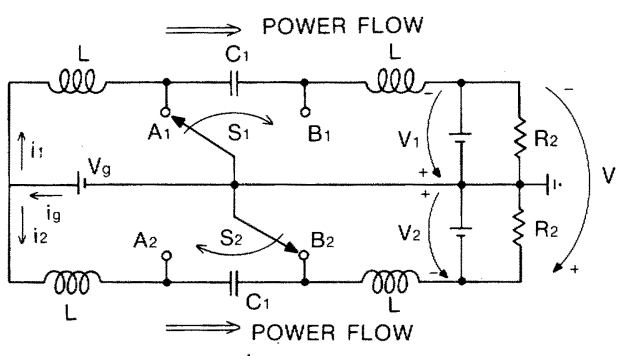

Fig. 16. Two bidirectional Cuk converters operating in parallel from a single power supply.

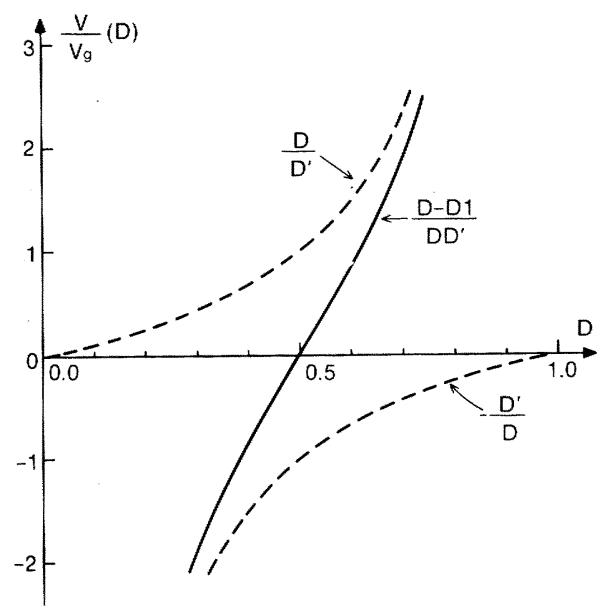

Fig. 17. Differential voltage gain $V / V_{s}$ for the power stage in Fig. 16.

voltage of either polarity, depending upon a switch duty ratio $D$. The design should preserve all the good properties of the new converter previously described and possibly add some more. Since the use of dual power supplies, as in the buck converter power amplifier, adds to the expense and complexity of the design, the additional feature of a single power supply is desired. Consideration of this requirement led to the discovery of the push-pull type topology shown in Fig. 16, in which two bidirectional switching converters are operated in parallel from a single supply.

Let us assume that the two converters are operated switching out of phase, that is, with complementary drive ratios. Namely, when switch $S_{1}$ is in position $A_{1}$ for interval $D T_{s}$, switch $S_{2}$ is in position $B_{2}$ for the same interval. Assuming normal operating conditions, the ideal voltage gain of the top stage is $D / D^{\prime}$, and that of the bottom is $D^{\prime} / D$. Thus, the two output voltages are equal only for a duty ratio of 0.5 , while one or the other becomes greater for other values of $D$. Evaluating the difference of the two output voltages $V=V_{1}-V_{2}$ gives a differential voltage gain of

$$
\frac{V}{V_{g}}=\frac{D-D^{\prime}}{D D^{\prime}}
$$

which is plotted as a function of duty ratio $D$ in Fig. 17 (heavy line). The individual converter gains are shown as dotted lines.

As seen in the figure, the differential gain is just the one needed for a switching power amplifier, since it has the same required polarity change property as that of the modified buck power stage (Fig. 11). The only trouble, however, is that the load is not across the two converter outputs. Thus, an interest- 


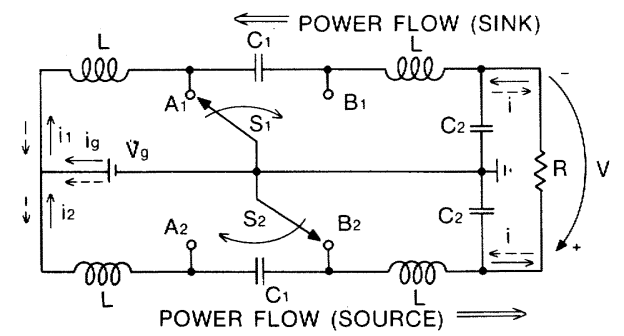

Fig. 18. The Cuk push-pull switching power stage.

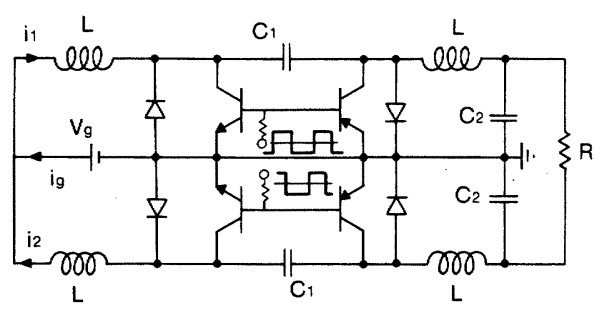

Fig. 19. Hardware implementation of the Cuk power stage.

ing question arises. Is it possible to connect a load between the two outputs without violating any basic circuit laws or disturbing the proper operation of the converters?

The answer to this question is affirmative, and is a key to the success of the new push-pull. switching power amplifier design. With the two loads in the converter of Fig. 16 replaced by a differential ("floating") load $R$, the new push-pull power stage of Fig. 18 is obtained.

In Fig. 16, each converter operates independently, with unidirectional current and power flow as shown. However, this is not so in the new push-pull power stage. Owing to the differential load, current originating from one converter must be "sunk" by the other, resulting in equal and opposite current flow, as shown by the solid arrows in Fig. 18. With the opposite polarity of the output current, (dotted lines) the roles are reversed. Thus, the switches $S_{1}$ and $S_{2}$ must permit this bidirectional flow, depending upon the duty ratio $D$. In other words, a part of the energy delivered by one converter is consumed by the load, and the remainder returned by the other to the source. This bidirectional flow is easily accomplished, as discussed previously, and as shown in Fig. 19.

It may now become evident that the new amplifier power stage may be called a true push-pull power stage. Namely, while the lower converter pushes the current (and energy) through the load, the upper converter pulls if from the load and vice versa. This is quite unlike the conventional push-pull class B linear amplifiers for which a push-pull configuration would be a more appropriate designation.

In addition to the advantages of the bidirectional new converter already described, another very desirable feature derives from this true push-pull configuration itself. Note that since one converter operates as a power sink, the current drawn from the source by the other is reduced by the return current ( $i_{2}$ in Fig. 18) which is increasing when $S_{2}$ is in position $B_{2}$ as shown. (The capacitor is discharging return power into the power supply inductor of converter 2.) Since current $i_{1}$ is increasing at the same time, the current ripple through the power supply is reduced. In fact, if the two inductors on the supply side are equal, the current drawn from the power supply is pure dc with no ripple at all.

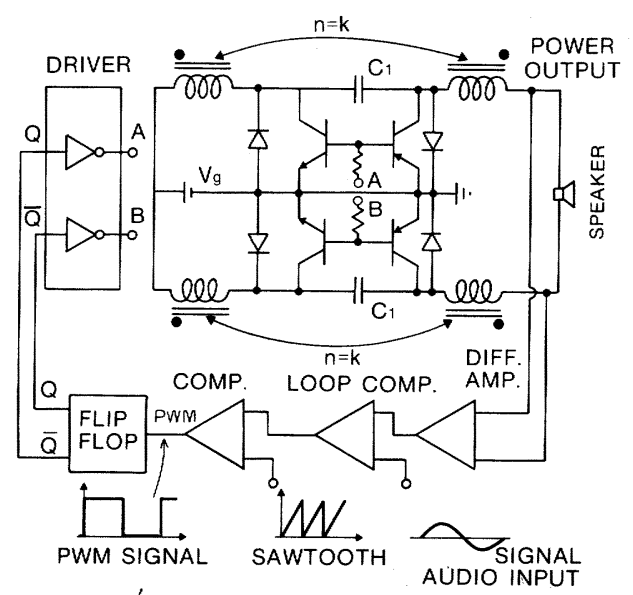

Fig. 20. Cuk push-pull switching power amplifier.

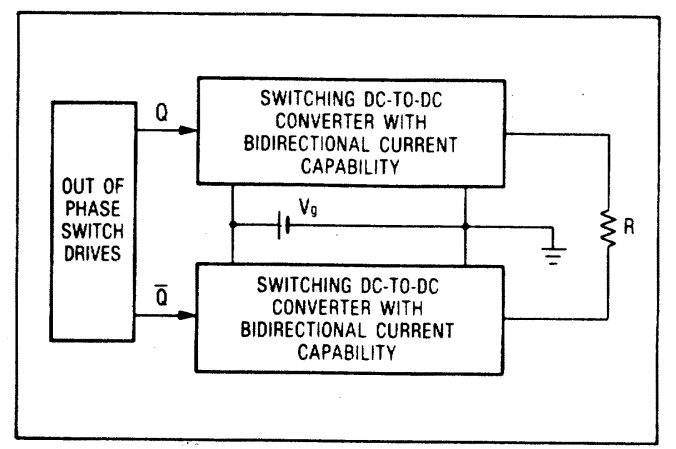

Fig. 21. New generalized concept of constructing a switching amplifier power stage from any dc-to-dc converter type.

The most advantageous configuration is obtained, however, when the coupled-inductor extension of the power stage in Fig. 19 is used. The resulting design is shown in Fig. 20, which also represents a complete block diagram of a closed-loop switching power amplifier using the new push-pull configuration. When the two transformers (coupled-inductors) are designed to satisfy the matching condition, as described earlier, the output current ripple, and consequently the need for output capacitors, is completely eliminated. Removing the capacitors results in extremely favorable phase-frequency response and permits closing the feedback loop directly, even without any compensation network, and yet with a high degree of stability. Also, there is no longer any need for an excessively high switching frequency to reduce output ripple, thus resulting in further improvement.

It should be emphasized that the new switching amplifier of Fig. 19 is actually based on a new concept of significantly broader scope. Namely, this novel technique of connecting a single dc source to a true push-pull configuration with a differentially connected load may be implemented using any other converter type, as illustrated in Fig. 21, provided that the converters are capable of bidirectional current flow and are driven with complementary (out of phase) switching drives [10]. In particular, when this configuration is implemented using two buck-type converters, a single-source power amplifier is obtained even with the buck power stage.

\section{Performance of the New Converter and Amplifier}

The new switching amplifier design retains all of the optimal characteristics of the new converter topology, while simul- 


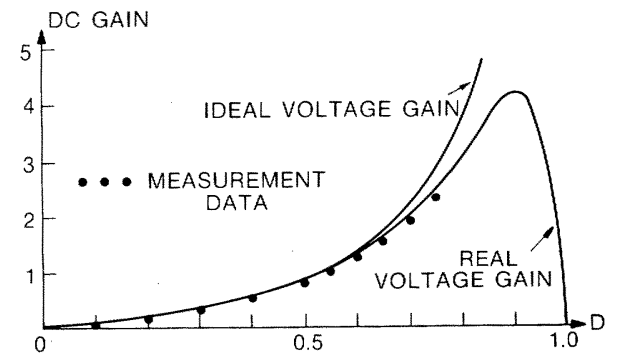

Fig. 22. Theoretical and actual dc voltage gain characteristics of a typical test circuit. Experimental measurements confirm the predicted gain curve.

taneously offering improvements due to the push-pull configuration itself. However, one important property of the new design, the linearity of the voltage gain, has not been discussed. Note that although the gain curves of the individual converters in the new design are highly nonlinear, as shown by the dotted lines in Fig. 17, the differential voltage is very linear near the duty ratio $D=0.5$, just where it is needed the most-in the center of its useful dynamic range. Nonetheless, sources of distortion, as well as the effects of nonideal circuit elements, must now be addressed to more accurately characterize the performance of the new power stage.

The new converter design has been extensively analyzed and experimentally verified. Limitations to the theoretical $\mathrm{dc}$ voltage gain arise due primarily to the effects of the parasitic resistance in the windings of the input and output inductors. Consideration of these terms in the analytical circuit model leads to a predicted voltage gain curve of

$$
\frac{V}{V_{g}}=\frac{D}{D^{\prime}}\left[\frac{1}{1+a_{1}\left(\frac{D}{D^{\prime}}\right)^{2}+a_{2}}\right]
$$

where $a_{1}$ and $a_{2}$ are the ratios of the series resistances of the input and the output inductors to the load resistance, respectively, and $R$ is the equivalent resistance of the load. A plot of this curve for a test circuit is shown in Fig. 22, and conforms well to the measured experimental results.

The current gain is unaffected and remains equal to $D^{\prime} / D$ as before. Thus, the power conversion efficiency for a given duty ratio is equal to the bracketed term in the above expression. As the duty ratio approaches one, the power dissipated in the inductor grows significant and reduces the operating efficiency of the circuit. The power loss due to the output resistance is independent of the operating point, and becomes the most significant detrimental factor when $D$ is less than 0.5 (voltage reduction).

This model does not consider the effects of other resistive losses that occur because of nonzero voltage drops across the semiconductors (when operating) and the finite switching time they require to become fully conducting. These losses may become significant in applications involving relatively low input or output voltages or high switching frequencies. Nevertheless, all these effects can easily be included analytically by use of the modelling methods described in [7] .

When the effects of the parasitic resistances on the performance of the push-pull power stage are included, the differential gain curve of Fig. 23 results. As mentioned earlier, the curve

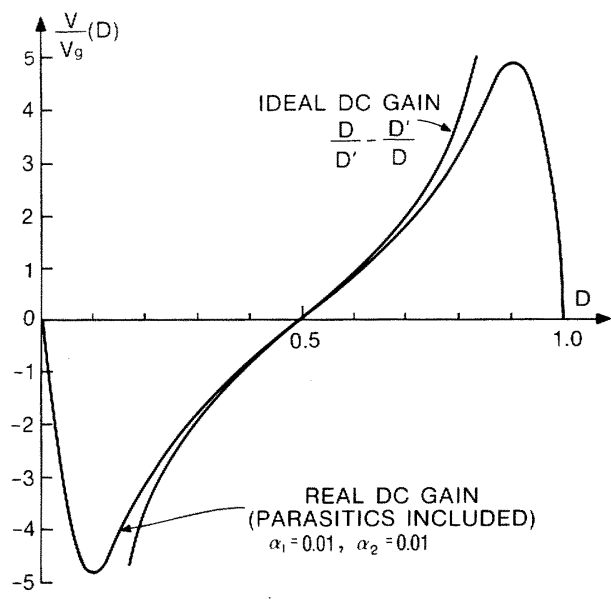

Fig. 23. Effective differential gain curve when parasitic resistances are considered.

for the ideal case (both $a_{1}$ and $a_{2}$ zero) is fairly linear about the center of the operating region $D=0.5$. For example, if the variation of the duty ratios in the PWM signal is limited to \pm 0.1 (around $D=0.5$ ), the resulting total harmonic distortion (THD) in the output will be limited to only 1 percent [8] . However, a variation of \pm 0.2 produces 4 -percent distortion, and increasingly higher THD values result from broader excursions.

Furthermore, as can be seen from the figure, the parasitic resistances tend to further linearize the gain around $D=0.5$. In fact, the values of these resistances can be adjusted to minimize the distortion over the entire operating region. By adding a small resistance in series with the input inductor so that the value of $a_{1}$ matches the optimality criterion $a_{1}=0.0714 \mathrm{X}$ $\left(1+Z a_{2}\right)$, THD can be reduced to well below 0.1 percent over the range $D=0.5 \pm 0.2[8]$. Thus, almost perfect linearity in the dc gain curve is achieved even without the use of feedback. Although adding an input resistor results in slightly degraded efficiency, test circuits still perform in the 90-percent range if the variation in the duty ratio is suitably limited. This need pose no unreasonable restriction, however, if the transformerisolated version of the converter is used [4].

Equally significant is the effect of the series resistance on the frequency response of the new converter circuits. Although each converter stage has three energy storage elements, which could theoretically introduce complications due to multiple resonances, the damping provided by the input resistance effectively produces a highly favorable "single-pole" frequency response [8]. This completely eliminates feedback stabilization problems and allows closing the loop without a compensation network, greatly simplifying design and resulting in even further savings in circuit size, weight, and cost.

\section{CONCLUSIONS}

We have shown how a fundamentally new design in switching regulator circuit topology has resulted in dramatically improved performance. The new converter not only offers higher efficiency, low or zero output ripple, and greatly reduced EMI, but at the same time achieves the general conversion function-it is capable of either increasing or decreasing the output voltage depending upon the duty ratio of the switching transistor. The new topology uses capacitive energy transfer between 


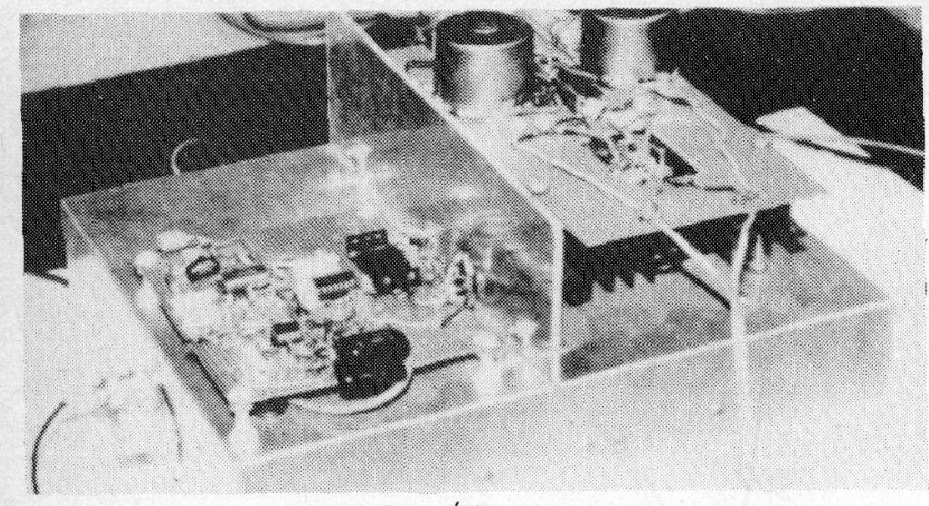

Fig. 24. Prototype version of a Cuk switched mode audio power amplifier. The circuit delivers $40 \mathrm{~W}$ rms to an $8 \Omega$ speaker load, with a flat frequency response over the $20-\mathrm{Hz}-20 \mathrm{KHz}$ range, and over 90 percent efficiency. A recently built model has been reduced to less than one quarter this size.

the input and output stages rather than the inductive energy transfer of other converters, resulting in nonpulsating input and output current. Its implementation requires fewer parts and simpler drive circuitry, with attendant savings in circuit size, weight, and cost.

The excellent frequency response characteristics of the new design allow highly stable feedback regulation to be achieved with simple circuitry. As a result, the basic converter may be used in a new switching power amplifier design that offers performance comparable to linear amplifiers but at much higher efficiency and lower cost-offering, for the first time, efficient dc-to-ac power conversion.

Thus, the new optimum topology converter is superior to any of the currently known switching converters, out performing them in every respect (Fig. 24).

\section{APPENDIX \\ A GENERAL AND UNIFIED APPROACH TO POWER CONVERTER TOPOLOGY}

\section{A. Modular Concept}

A large number of switching configurations are currently available for a multitude of power conversion functions. Heretofore, some circuit configurations have been used solely to perform the dc-to-dc conversions function (converters), others for dc-to-ac conversion (inverters), and yet another for power amplificiation, with their circuit configurations having nothing or very little in common. With the family of Ćuk converters, however, a vertical integration has been made, and for the first time a logical extension from a single-quadrant to a two-quadrant converter (battery charger/discharger) and finally to a four-quadrant converter (bidirectional power amplifier) has been realized.

\section{B. Quadrant Classification}

Power processing systems may be classified according to the nature of their output capabilities. The simplest is a dc-to-dc converter in which output current is delivered in one direction at one output voltage polarity. This means that on a graph of output voltage $V$ versus output current $I$, only one quadrant is available, as shown for positive $V$ and $I$ in Fig. 25(a). This is structurally the simplest switched-mode converter, and can be realized with only one transistor and one diode, as in the Cuk converter illustrated.
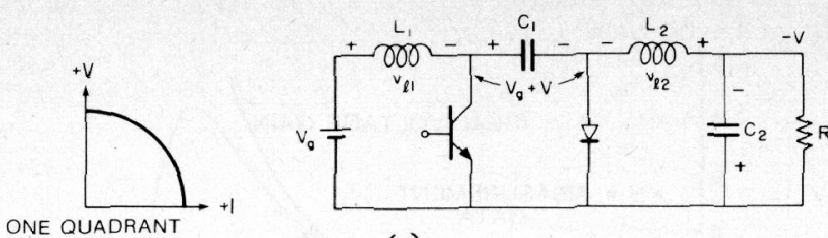

(a)
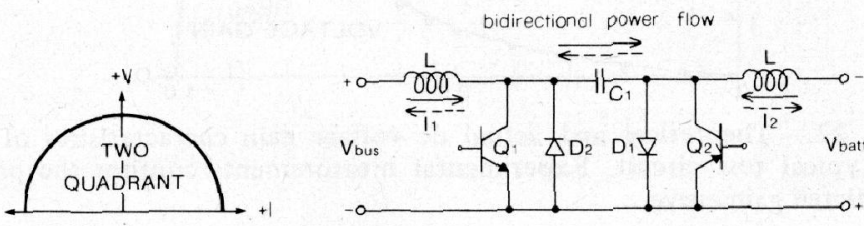

(b)
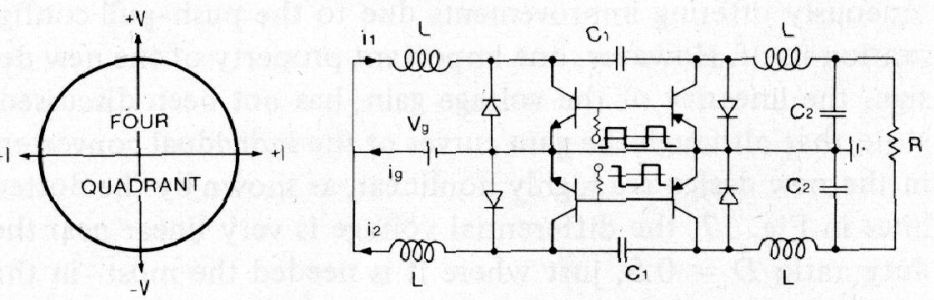

(c)

Fig. 25. (a) Basic Ćuk converter with one-quadrant classification. (b) Battery charger/discharger with two-quadrant classification. (c) Bidirectional power amplifier with four-quadrant classification.

Simply by doubling the number of power devices in the Cuk converter of Fig. 25(a), the output capability of a dc-todc converter can be extended so that current can flow in either direction (bidirectional current switch implementation), and the system becomes a two-quadrant converter as shown in Fig. 25(b). Thus, INPUT and OUTPUT can be arbitrarily interchanged, and the converter is capable of bidirectional power flow.

Finally, by the new general concept of converter topological interconnection illustrated in Fig. 21, a two-quadrant converter is extended into a four-quadrant converter in which the directions of both voltage and current can change independently as shown in Fig. 25(c). Hence, a true ac output can be obtained together with bidirectional power flow.

Although this vertical integration has been conceived for the family of Cuk converters, it can be directly applied to other converter types, such as buck, boost, or any other switching configuration.

\section{Applications}

The Cuk converters have a broad range of applications in all aspects of power processing. Robotics, in particular, will benefit from the excellent performance of Cuk converters in motor control. Its high efficiency and low noise make it an obvious choice as a dc motor servo drive, with regenerative braking, in either the two- or four-quadrant configurations. In fact, the high performance of the Cuk bipolar amplifier makes it possible to design servo-systems employing ac induction motors, capable of optimum motor drive, as well as regenerative braking.

\section{ACKNOWLEDGMENT}

Special thanks go to R. Erickson and W. Behen, members of the Power Electronics Group at the California Institute of Technology, for many devoted hours spent in building the first 
prototype of the new switching power amplifier and the later improvements.

\section{REFERENCES}

[1] Slobodan Cuk and R. D. Middlebrook, "A new optimum topology switching dc-to-dc converter," in 1977 IEEE Power Elec. Specialists Conf. Rec., pp. 160-179.

[2] Slobodan Cuk, "Modelling, analysis, and design of switching converters," Ph.D. thesis, California Inst. Technology, Nov. 1976. Also, NASA Rep. CR-135174.

[3] Slobodan Cuk and R. D. Middlebrook, "Coupled-inductor and other extensions of a new optimum topology switching dc-to-dc converter," in 1977 IEEE Industry Applications Soc. Ann. Meet. Rec., pp. 1110-1126.

[4] R. D. Middlebrook and Slobodan Ćuk, "Isolation and multiple output extensions of a new optimum topology switching dc-to-dc converter," in 1978 IEEE Power Electronics Specialists Conf. Rec., pp. 256-264.

[5] R. D. Middlebrook, Slobodan Cuk, and W. Behen, "A new battery charger/discharger converter," in 1978 IEEE Power Electronics Specialists Conf. Rec., pp. 251-255.

[6] L. Shaeffer, "VMOS-A breakthrough in power MOSFET technology," Siliconix Applications Note AN76-3, Santa Clara, CA 1976.

[7] R. D. Middlebrook and Slobodan Cuk, "Modelling and analysis methods for dc-to-dc switching converters,"' (invited review paper) in 1977 IEEE Int. Semiconductor Power Converter Conf. Rec., pp. 90-111.

[8] Slobodan Cuk and Robert W. Erickson, "A conceptually new highfrequency switched-mode amplifier technique eliminates current ripple,"' in Proc. Fifth Nat. Solid-State Power Conversion Conf., May 1978, pp. G3.1-G3.22.

\title{
Advances in Switched-Mode Power Conversion Part II
}

\author{
SLOBODAN ĆUK, MEMBER, IEEE, AND R. D. MIDDLEBROOK, FELLOW, IEEE
}

\begin{abstract}
A number of important practical extensions to the basic Cuk converter are presented. They include dc isolation, multipleoutput power sources, and a physical realization of the sought for hypothetical dc-to-dc transformer, a device which converts from pure dc (no voltage or current ripple) at one terminal, to pure dc (at a different voltage) at the other terminal. The application of the circuit in a highly efficient amplifier for the servo control of a de motor or other loads is also presented.
\end{abstract}

\section{INTRODUCTION}

$\mathbf{I}_{\mathrm{t} y}^{\mathrm{N}}$ PART I of this series we provided a review of the basic types of switched-mode power converters and showed how the effort to solve the characteristic problems of these earlier designs led to the development of a fundamentally new converter configuration. Fig. 1 shows a summary of the basic converter types and a physical realization of the new converter in its simplest form. The new converter topology embodies all of the desirable features of previous types while retaining none of their liabilities. Whereas other converters rely upon the inductive coupling of energy between the input and output, the new converter uses a capacitor to transfer stored energy between input and output inductors as shown.

Due to the presence of inductors on both the input and output, the current on either terminal remains continuous

Manuscript received October 15, 1982.

The authors are with the California Institute of Technology, Pasadena, CA 91125.

This article is reprinted from Robotics Age Magazine. avoiding the electrical noise problems associated with switching either the input or output current and resulting in increased conversion efficiency. The new converter has the desirable property that its output voltage can be either higher or lower than that of the input supply, as determined by the duty ratio of the switching transistor (the fraction $D$ of the switching period $\left(T_{s}\right)$ that the transistor is turned on).

Also presented in Part I were several important extensions to the basic converter design. These include adding the capability for bidirectional power flow between input and output, coupling the input and output inductors via a single transformer core, and a high-performance switched-mode power amplifier configuration using parallel converters driving a differentially connected load.

An important result of the coupled-inductor extension of the new converter, apart from the further reduction in the number of components, is that, by proper adjustment of the magnetic coupling between the input and the output inductors, the residual current and ripple at one of the terminals can be reduced exactly to zero, resulting in pure dc. Naturally, this motivated the search for a converter configuration which would achieve the desired characteristic of having zero current ripple at both input and output simultaneously, thus resulting in a physical realization of an ideal dc-to-dc "transformer." (See Fig. 8 in Part I.)

In this article, we will show how, by pursuing the desirable property of $\mathrm{dc}$ isolation between the input and the output cir- 